\title{
Adaptable Photochromic Switches with Self-Aggregating Heterocyclic Azo Dyes
}

\author{
Jaume Garcia-Amorós, ${ }^{\dagger}$ M. Cidália R. Castro, ${ }^{\ddagger}$ Santi Nonell, ${ }^{\S}{ }^{\odot}$ Susana Vílchez, ${ }^{\|}$Jordi Esquena, ${ }^{\| \odot}$

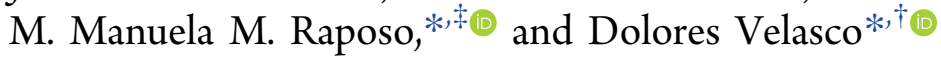

${ }^{\dagger}$ Grup de Materials Orgànics, Institut de Nanociència i Nanotecnologia (IN2UB), Departament de Química Inorgànica i Orgànica (Secció de Química Orgànica), Universitat de Barcelona, Martí i Franquès 1, E-08028 Barcelona, Spain

¥Centro de Química, Universidade do Minho, Campus de Gualtar, R. da Universidade, 4710-057 Braga, Portugal

${ }^{\S}$ Institut Químic de Sarrià, Universitat Ramón Llull, Via Augusta 390, E-08017 Barcelona, Spain

"Institute of Advanced Chemistry of Catalonia, Spanish National Research Council (IQAC-CSIC) and Networking Research Center on Bioengineering, Biomaterials and Nanomedicine (CIBER-BBN), E-08034 Barcelona, Spain

\section{Supporting Information}

ABSTRACT: It is well-known that the thermal isomerization kinetics of photochromic azo dyes can be modulated by subtle changes in their chemical architecture. However, the availability of an orthogonal input to control the thermal relaxation of azo dyes is essential to enable access to multifunctional and adaptive photochromic switches based on these particular organic chromophores. In this work, we have designed and synthesized a new family of green-light-activated heterocyclic azo derivatives that modify their switching capabilities as a function of concentration. In this line, we have investigated their self-assembly and the nature of the supramolecular aggregates formed by means of dynamic light scattering, polarized optical microscopy, and X-ray diffraction.

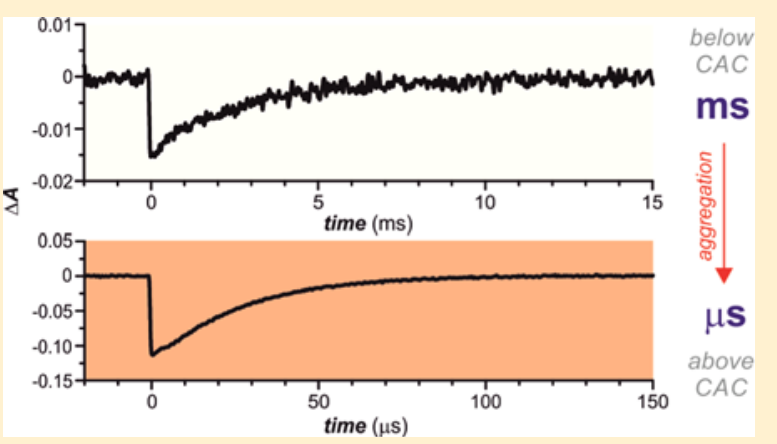
Indeed, imparting control over the self-assembly of these organic dyes allows to fine-tune their thermal relaxation time and produce adaptable photochromic switches. Specifically, swapping the azo dye concentration between values located above and below the corresponding critical aggregation concentration modifies significantly the relaxation time up to 250 times, i.e., from the millisecond to the microsecond timescale. Moreover, the optical density of the system can be switched back and forth hundreds of times, for both diluted and concentrated solutions, without any sign of fatigue.

\section{INTRODUCTION}

The rapid development of current science and technology is promoting, more than ever, the advent of novel dynamic stimuli-responsive materials capable of performing complex tasks on demand. In this context, the design and study of switchable artificial molecular systems and supramolecular assemblies has been garnering a great deal of attention during recent years as a fundamental step for the generation of adaptable materials with emergent behaviors and abilities.

Among all possible input energies, light is the trigger of common choice for switching purposes since it permits not only the remote and noninvasive regulation of distinct physiological events but also the precise modulation of the macroscopic properties of diverse synthetic systems, thereby giving rise to a new generation of materials with attractive properties. $^{1-9}$ These features explain the increasing popularity of photoswitches.

Azobenzene derivatives are probably the most used organic photochromes for switching applications owing to their relatively straightforward synthesis, tunable wavelength, and high photostability. Repeatable actuation is usually achieved with these dyes by the interplay of two light sources operating at different wavelengths; in the case of unsubstituted azo dyes UV and blue light, which trigger the trans-to-cis and cis-to-trans isomerization, respectively. ${ }^{10}$ However, the thermal regeneration of the trans isomer is generally preferred since it avoids the use of a second external stimulus and diminishes the complexity of the final material.

The relaxation time of the metastable cis form can range from a few nanoseconds to several years and is, therefore, a benchmarking criterion for the ultimate applicability of the system. ${ }^{11-21}$ In fact, while the photoinduced trans-to-cis isomerization kinetics occurs within the picosecond timescale and depends mainly on the intensity of the excitation beam, the thermal back reaction is governed essentially by the molecular architecture of the azo photochrome. On this basis, tuning the thermal stability of the cis isomer implies careful, subtle and, most importantly, irreversible structural modifica-

Received: August 7, 2019

Revised: August 27, 2019

Published: August 28, 2019 
tions. In this context, the availability of an orthogonal input to control the thermal relaxation of azo dyes would enable access to multifunctional and adaptive photochromic switches based on these particular organic chromophores. Self-assembly might lead to dramatic changes in the thermal isomerization rate of azo photochromes. To the best of our knowledge, there is only one previous report about the influence of self-aggregation on the thermal isomerization kinetics of a relatively slowisomerizing azo- $\mathrm{BF}_{2}$ complex. ${ }^{22}$ This behavior has been also studied for other organic chromophores such as merocyanines $^{23}$ and dithiafulvenes. ${ }^{24}$

Herein, we demonstrate how self-aggregation can be exploited for the active tuning of the thermal isomerization rate of fast azo dyes. Specifically, we report on the design, synthesis, photochromism, and self-assembly of a novel series of green-light-activated heterocyclic azo compounds, which modify their isomerization rate significantly due to the formation of supramolecular aggregates. This finding furnishes a new external input for controlling the kinetics of the thermal back reaction of azo derivatives.

\section{RESULTS AND DISCUSSION}

For the present study, two different azo photochromes have been devised by pairing the powerful electron-donating bithienylpyrrole (Tph-Tph-Pyr) triad with carboxyl or formyl electron-withdrawing groups within the very same covalent skeleton in the shape of $\mathbf{1}$ and $\mathbf{2}$ (Scheme 1). In addition, the

\section{Scheme 1. Chemical Structure of Heterocyclic Azo} Derivatives 1-5

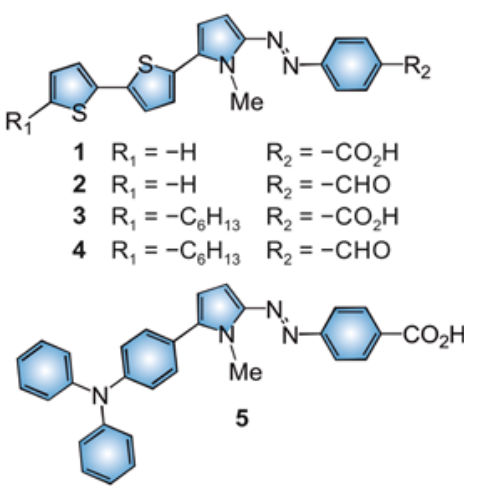

introduction of either flexible lateral alkyl chains (compounds 3 and 4) or non-rodlike electron-donating moieties (compound 5) has been also considered to study its influence on the organization of the system at the supramolecular level and, in turn, on its thermal cis-to-trans isomerization kinetics.

Bithienylpyrrole precursor 6 was prepared using the synthetic methodology reported by us recently. ${ }^{25}$ Novel bithienylpyrrole 7 and pyrrolyldiphenylaniline 8 precursors were synthesized through a Suzuki cross-coupling reaction between the commercially available 1 -methyl- $1 \mathrm{H}$-pyrrol-2-yl-2boronic acid and either 5-bromo-5-hexyl-2,2'-bithiophene or 5bromo- $N, N$-diphenylaniline in 1,2-dimethoxyethane in good to excellent yields (83-96\%; Scheme S1). Diazotation of 4aminobenzaldehyde and 4-aminobenzoic acid with $\mathrm{NaNO}_{2}$ in $\mathrm{HCl}$ at $0-5{ }^{\circ} \mathrm{C}$ afforded the corresponding diazonium salts, which were further reacted with bithienylpyrroles $6-7$ and pyrrolyldiphenylaniline 8 in methanol and pyridine at $0-5{ }^{\circ} \mathrm{C}$ to give heterocyclic azo dyes $\mathbf{1 - 5}$ in moderate to excellent yields (36-94\%; Schemes S2 and S3). Electrospray ionization mass spectrometry together with ${ }^{1} \mathrm{H}$ and ${ }^{13} \mathrm{C}$ NMR spectra confirmed their structural identities.

The azo derivatives prepared absorb significantly along the visible region of the electromagnetic spectrum. Specifically, the absorption spectra (Figure 1) of $\mathbf{1 - 5}$ in ethanol at $298 \mathrm{~K}$

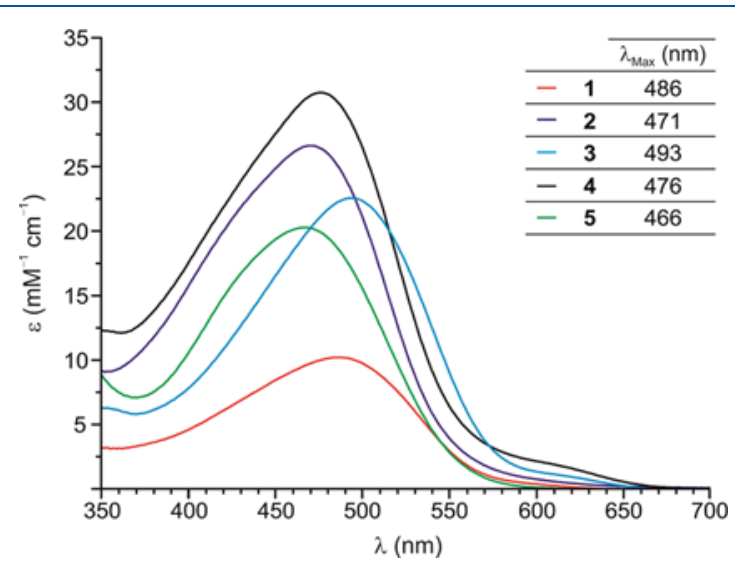

Figure 1. Absorption spectra for azo dyes $\mathbf{1 - 5}$ in ethanol at $298 \mathrm{~K}$.

shows a main absorption band peak between 466 and $493 \mathrm{~nm}$ $\left(\lambda_{\max }\right.$ in Figure 1$)$, with molar absorption coefficients $(\varepsilon$ in Figure 1) ranging from 10 to $31 \mathrm{mM}^{-1} \mathrm{~cm}^{-1}$. This strong absorption can be ascribed to the overlapping of the $\pi-\pi^{*}$ and $\mathrm{n}-\pi^{*}$ transitions of the heterocyclic chromophore. Pulsed irradiation of the $\pi$-conjugated system with green light $\left(\lambda_{\text {ex }}=\right.$ $532 \mathrm{~nm}$ ) results in a decrease in absorption at $\lambda_{\mathrm{obs}}$ due to the photoinduced trans-to-cis isomerization of the $\mathrm{N}=\mathrm{N}$ bond. After the laser pulse, the corresponding pre-irradiation absorbance value is restored completely because of the cis-totrans thermal back reaction. The kinetics of the process has been monitored by means of nanosecond laser flash photolysis (Supporting Information) in ethanol at $298 \mathrm{~K}$. In all instances, the thermal relaxation time $(\tau=1 / k)$ of the $c i$ isomers has been determined by fitting a monoexponential function to the data. The concentration dependence of $\tau$ has been also studied to investigate the impact of the self-aggregation of the azo dye on this kinetic parameter.

As a whole, the thermal switching rate of azo dyes 1-4, which contain the rodlike Tph-Tph-Pyr triad within their covalent skeleton, falls within the milli- and microsecond temporal domain and depends significantly on the amount of chromophore present in the alcoholic solution (Figure 2). cis5, however, shows a thermal relaxation time within the millisecond timescale $(10.5 \mathrm{~ms})$ due to the poorer electrondonating character of the pyrrolyldiphenylaniline platform, which remains essentially constant within the whole range of concentrations analyzed.

The concentration dependence of the relaxation time for the cis form of azo derivatives 1-4 (Figure 3) reveals two welldefined linear regimes with different slopes. From the intercept of the two fits, the critical aggregation concentration (CAC in Figure 3) value is estimated. Within the former region, which comprises concentration values higher than the CAC, the system exhibits a very fast and slightly varying isomerization rate. This behavior can be well-understood based on all azo molecules being assembled at the supramolecular level, forming stable aggregates. As a representative example, a 1.7fold increase in concentration from 23 to $38 \mu \mathrm{M}$ reduces the 


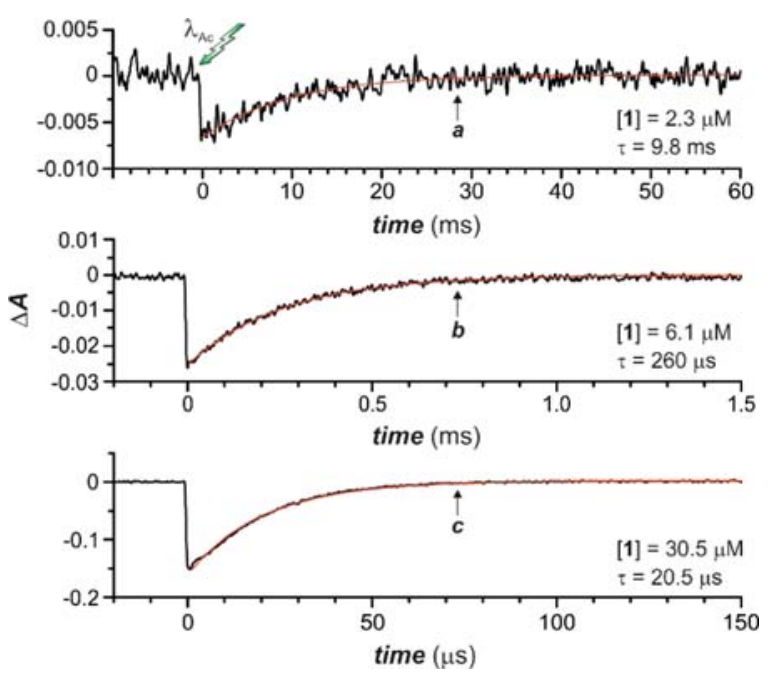

Figure 2. Transient absorption change photoinduced by laser-pulsed irradiation with green light (Nd-YAG laser, $5 \mathrm{~ns}$ pulse width, $10 \mathrm{~mJ}$ per pulse, $\lambda_{\text {ex }}=532 \mathrm{~nm}, \lambda_{\text {obs }}=510 \mathrm{~nm}$ ) for azo dye 1 in ethanol at $298 \mathrm{~K}$ at different concentrations: $2.3 \mu \mathrm{M}$ (a), $6.1 \mu \mathrm{M}$ (b), and 30.5 $\mu \mathrm{M}(\mathrm{c})$.

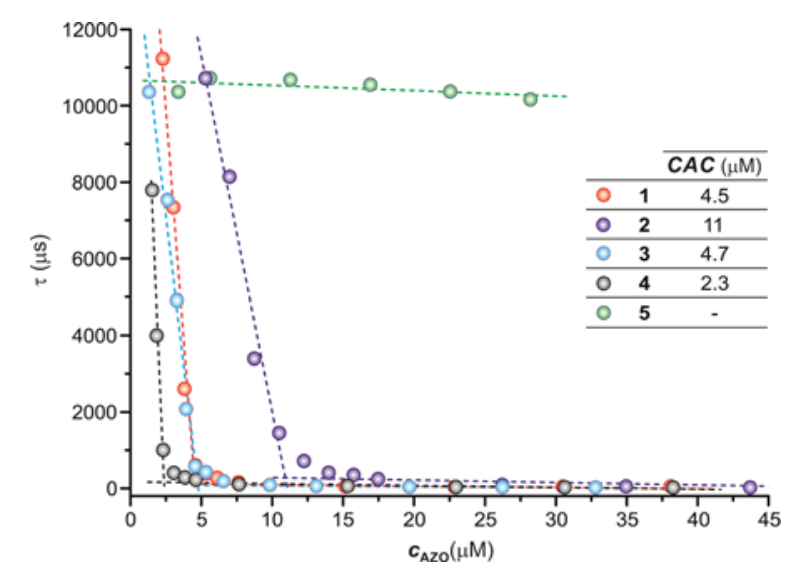

Figure 3. Concentration dependence of the relaxation time, $\tau$, in ethanol at $298 \mathrm{~K}$ for the thermal cis-to-trans isomerization of azo dyes 1-5. The critical aggregation concentration (CAC) for each azo dye is also shown.

relaxation time of 4 by a factor of only 1.5 , from 34.6 to 22.5 $\mu$ s, under ambient conditions. Within the second region, i.e., at concentrations below the CAC, a significant variation of the thermal isomerization rate of the azo derivative with the concentration is observed (Figure 3). In this case, a 2 -fold increase in the concentration of 4 from 1.5 to $3.1 \mu \mathrm{M}$ reduces the relaxation time of the cis form by a factor of 19.2 , from 7.8 $\mathrm{ms}$ to $404 \mu \mathrm{s}$. On the basis of these considerations, the thermal switching rate of the system can be easily modulated by controlling the concentration of the azo photochrome and, in turn, its self-assembly at the supramolecular level. Certainly, the highest variations are detected when swapping the concentration of the azo compound between values located above and below the corresponding CAC. This switching cannot be achieved with the pyrrolyldiphenylaniline-based azo dye $\mathbf{5}$, thus limiting its further applicability.

Self-aggregation of the azo molecules in ethanol has been explored by dynamic light scattering (DLS) and polarized optical microscopy (POM). For these experiments, concentrated solutions $(0.3-0.6 \mathrm{mM})$ of azo dyes $1-5$ were used to obtain detectable light scattering signals and suitable POM microphotographs. On the one hand, azo dyes 1 and 3, which contain a carboxylic acid and the rodlike Tph-Tph-Pyr triad in their molecular structure, precipitate as micrometric crystals from ethanol at such a high concentration (Figure S1) independent of the presence of a flexible hexyl chain in the Tph-Tph-Pyr platform. More specifically, both 1 and 3 form short rodlike microparticles, which display birefringence under polarized visible light (Figure S1a,b). This anisotropy can be attributed to the crystalline nature of the microparticles. At higher magnification (Figure S1c,d), it can be observed that azo dye 1 forms larger rods, probably due to growth of initial particles. The crystalline nature of these two azo dyes was confirmed by X-ray diffraction (XRD) of dried powders. The XRD spectra of $\mathbf{1}$ and 3 (Figure S2a,b) indicate highly crystalline materials that produce a complex diffraction pattern. On the other hand, azo dyes 2,4 , and 5 did not precipitate and were analyzed by DLS, obtaining autocorrelation functions in these three cases (Figures 4 and S3). The autocorrelation
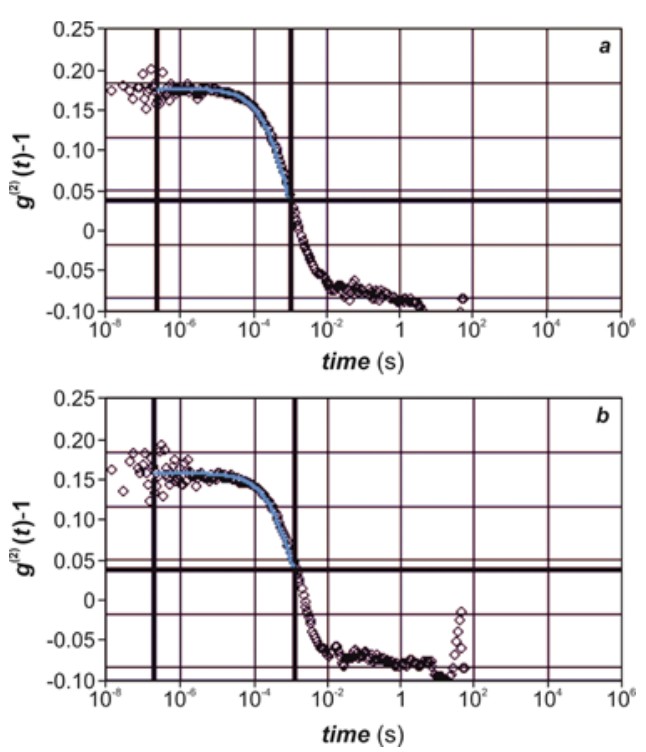

Figure 4. Second-order autocorrelation functions for azo dye 4 in ethanol at 46 (a) and $9.3 \mu \mathrm{M}(\mathrm{b})$, respectively, obtained by dynamic light scattering.

function provides information about translational diffusion, from which the hydrodynamic radius $\left(R_{\mathrm{h}}\right)$ is calculated by applying the Stokes-Einstein equation. DLS experiments conducted with solutions of 2 and 5 ( 0.52 and $0.34 \mathrm{mM}$, respectively) reveal that these two azo dyes self-assemble in ethanol, producing aggregates at the nanoscale, with their average hydrodynamic radii being 66 and $85 \mathrm{~nm}$, respectively. Furthermore, DLS evidences that azo dye 4 self-aggregates even at lower concentrations (9.3 and $46 \mu \mathrm{M})$. For these samples, with an azo dye concentration above CAC and falling within the concentration range considered in the kinetic analyses, hydrodynamic radii of 195 and $188 \mathrm{~nm}$ were obtained, respectively, indicating that an increase of the azo dye concentration in the alcoholic solution results in a large number of scattering units of a similar size. No DLS signals were detected with solutions of 4 at concentrations below CAC due to the very low presence of scattering units in such diluted samples. Thus, POM and DLS observations point out that all azo compounds aggregate in ethanol, forming either 

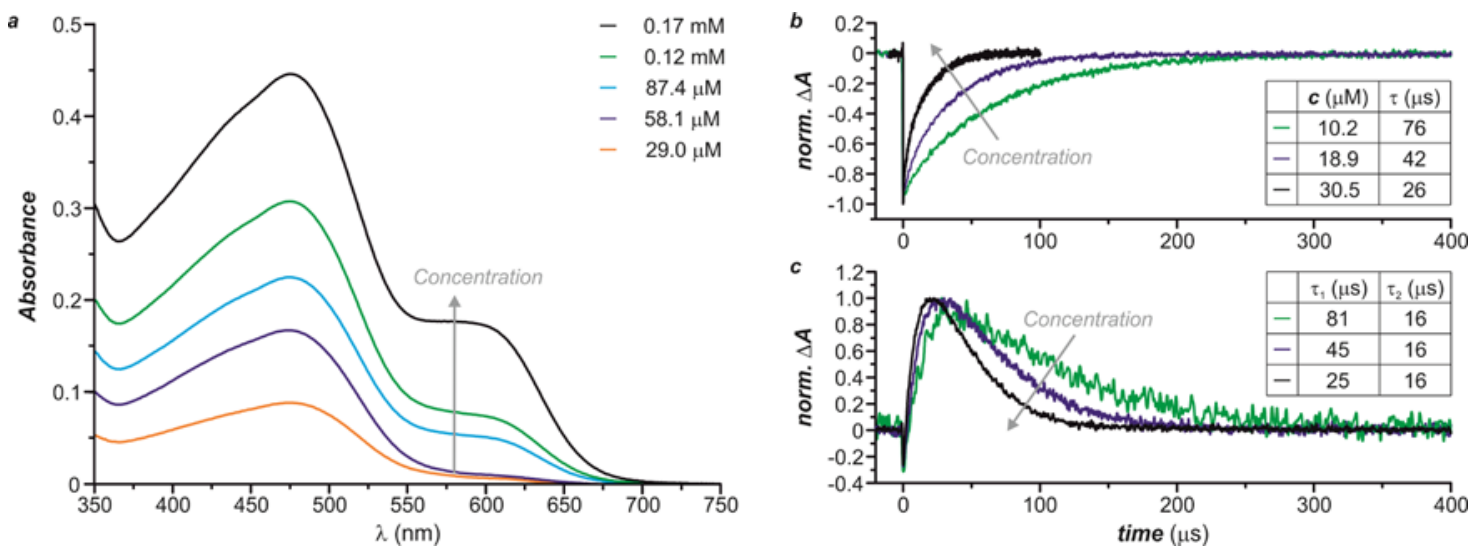

Figure 5. Evolution of the absorption spectrum with the azo dye concentration (a) and transient absorption change photoinduced by laser-pulsed irradiation with green light [Nd-YAG laser, $5 \mathrm{~ns}$ pulse width, $10 \mathrm{~mJ}$ per pulse, $\lambda_{\mathrm{ex}}=532 \mathrm{~nm}, \lambda_{\text {obs }}=500 \mathrm{~nm}(\mathrm{~b})$, and $\left.\lambda_{\text {obs }}=570 \mathrm{~nm}(\mathrm{c})\right]$ for azo dye 4 in ethanol at $298 \mathrm{~K}$.

nanoaggregates (dyes 2, 4, and 5 ) or microcrystals (dyes 1 and 3). However, while the CAC values for 1-4 fall within 2.3 and $11 \mu \mathrm{M}$ (Figure 3), the CAC value for 5 should be well below the limit of detection of our flash-photolysis setup.

The fact that all compounds aggregate can be ascribed to the effective $\pi-\pi$ stacking established in all the compounds analyzed. This effect might be more significant in compound 5, with a more $\pi$-extended system that enhances its aggregation. For azo compounds 1-4, which bear a rodlike Tph-Tph-Pyr triad within their covalent skeleton, two additional structural features can fine-tune their self-assembly, namely, the hydrogen-bonding capability and the establishment of van der Waals interactions between the lateral alkyl chains. A comparison between the concentration dependence of $\tau$ for azo derivatives $\mathbf{1}$ and $\mathbf{3}$ evidences that, in this case, the hydrogen bonding established between the carboxyl groups overcomes the effect of the alkyl chains, yielding the very same CAC for both azo dyes. This situation differs from the one observed for their formyl-based analogues $\mathbf{2}$ and 4, where the self-aggregation induced by the $\pi-\pi$ stacking is modulated by the presence of the lateral alkyl chain (CAC value of 11 and $2.3 \mu \mathrm{M}$ for 2 and 4, respectively).

To get more insight into the influence of supramolecular aggregation on the thermal cis-to-trans isomerization of this family of heterocyclic chromophores, we have investigated the evolution of the absorption spectrum and the distinct kinetic behavior at different observation wavelengths for azo dye 4 , as a representative example, with concentration. The absorption spectra collected at concentration values ranging from $29 \mu \mathrm{M}$ to $0.17 \mathrm{mM}$ show the appearance of an additional band centered at ca. $600 \mathrm{~nm}$ associated to the self-assembly of the azo photochromes (Figure 5a). The thermal isomerization of 4 has been analyzed at two $\lambda_{\text {obs }}, 500$ and $570 \mathrm{~nm}$, falling within the two main absorption bands. On the one hand, the kinetic trace obtained at $\lambda_{\text {obs }}=500 \mathrm{~nm}$ was nicely fitted to a monoexponential function (Figure $5 b$ ). However, the one registered at $\lambda_{\text {obs }}=570 \mathrm{~nm}$ followed a biexponential decay instead (Figure 5c). The first component $\left(\tau_{1}\right.$ in Figure $5 \mathrm{c}$ ) depends on the azo dye concentration and can be ascribed to its thermal isomerization process. It should be stressed that $\tau_{1}$ is in perfect agreement with the relaxation time detected at $\lambda_{\text {obs }}$ $=500 \mathrm{~nm}$ at each concentration. The second component $\left(\tau_{2}\right.$ in Figure 5c) does not depend on the azo dye concentration (constant $\tau_{2}$ value of $16 \mathrm{~ms}$ ), and we propose that it is related to the reorganization of the supramolecular aggregates after the trans-to-cis photoisomerization of the azo moities. This biexponential behavior is analogue to that detected by us for other azo dyes in nematic liquid-crystalline matrixes. ${ }^{14}$

The photostability of the different photochromic molecular switches has been tested by subjecting them to 100 pulsed green light $\left(\lambda_{\mathrm{ex}}=532 \mathrm{~nm}, 10 \mathrm{~mJ}\right.$ per pulse $)$ and dark cycles. Stability measurements have been performed at concentration values below and above the CAC of the corresponding azo dye. As it can be clearly seen in Figure 6, the system is perfectly photostable since neither the relaxation time nor the photoinduced absorption change upon irradiation get compromised after 100 switching cycles.

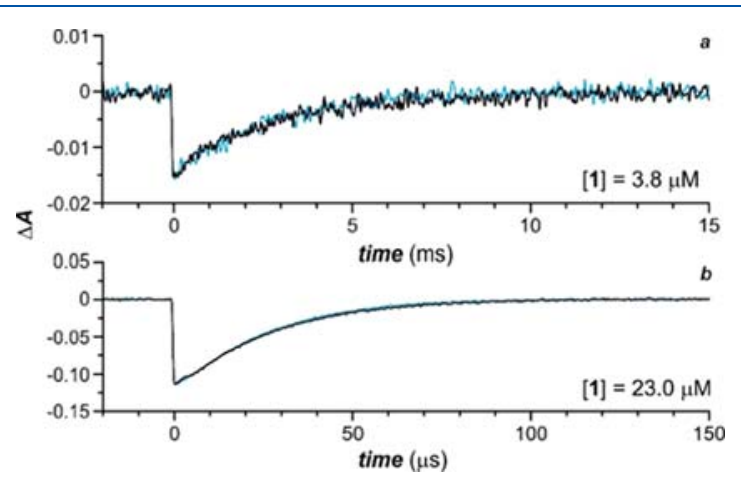

Figure 6. Transient absorption change photoinduced by laser-pulsed irradiation with green light (Nd-YAG laser, $5 \mathrm{~ns}$ pulse width, $10 \mathrm{~mJ}$ per pulse, $\lambda_{\text {ex }}=532 \mathrm{~nm}, \lambda_{\text {obs }}=510 \mathrm{~nm}$ ) for azo dye 1 in ethanol at $298 \mathrm{~K}$ before (black) and after (blue) 100 laser pulses at a concentration above $(3.8 \mu \mathrm{M},(\mathrm{a}))$ and below $(20.3 \mu \mathrm{M},(\mathrm{b}))$ the corresponding CAC value.

\section{CONCLUSIONS}

Heterocyclic azo switches possessing aldehyde or carboxyl electron-withdrawing groups and the Tph-Tph-Pyr triad as a $\pi$ conjugated and electron-donating system stand out as quick photochromic switches under ambient conditions. Remarkably, these organic photochromes self-assemble in alcoholic solutions producing notable variations in the observed thermal cis-to-trans isomerization kinetics. Specifically, the system changes its relaxation time from milliseconds to a few microseconds, i.e., more than 2 orders of magnitude, just by 
swapping the heterocyclic azo chromophore concentration below and above the corresponding CAC. Importantly, the replacement of the Tph-Tph-Pyr system by a non-rodlike pyrrolyldiphenylaniline platform results in a constant relaxation time over a wide range of concentrations. Herein, we have developed a new family of heterocyclic azo photochromes whose relaxation time can be finely tuned by means of two orthogonal external inputs, namely, illumination with green light and concentration of the chromophore in solution.

\section{ASSOCIATED CONTENT}

\section{S Supporting Information}

The Supporting Information is available free of charge on the ACS Publications website at DOI: 10.1021/acs.jpcc.9b07527.

Synthesis of the heterocyclic azo dyes; kinetic studies by nanosecond flash photolysis; characterization of the aggregation by polarized light optical microscopy, X-ray diffraction and dynamic light scattering (PDF)

\section{AUTHOR INFORMATION}

\section{Corresponding Authors}

*E-mail: mfox@quimica.uminho.pt (M.M.M.R.).

*E-mail: dvelasco@ub.edu (D.V.).

\section{ORCID}

Jaume Garcia-Amorós: 0000-0002-3554-7300

Santi Nonell: 0000-0002-8900-5291

Jordi Esquena: 0000-0002-9188-5259

M. Manuela M. Raposo: 0000-0002-7996-1626

Dolores Velasco: 0000-0002-9357-5860

\section{Notes}

The authors declare no competing financial interest.

\section{ACKNOWLEDGMENTS}

Financial support for this research was obtained from the Ministerio de Economía y Competitividad (Spain, PGC2018095477-B-I00, CTQ2016-78454-C2-1-R, and CTQ201784998-P MINECO/FEDER). Thanks are also due to Fundação para a Ciência e Tecnologia (Portugal) for financial support to the Portuguese NMR network (PTNMR, Bruker Avance III 400-Univ. Minho), FCT, and FEDER (European Fund for Regional Development)-COMPETEQREN-EU for financial support to the research centre CQ/UM [ref UID/ QUI/00686/2013 and UID/QUI/0686/2016], and a $\mathrm{PhD}$ grant to M.C.R.C. (SFRH/BD/78037/2011).

\section{REFERENCES}

(1) Beharry, A. A.; Woolley, G. A. Azobenzene photoswitches for biomolecules. Chem. Soc. Rev. 2011, 40, 4422-4437.

(2) Szymański, W.; Beierle, J. M.; Kistemaker, H. A. V.; Velema, W. A.; Feringa, B. L. Reversible photocontrol of biological systems by the incorporation of molecular photoswitches. Chem. Rev. 2013, 113, 6114-6178.

(3) Mart, R. J.; Allemann, R. K. Azobenzene photocontrol of peptides and proteins. Chem. Commun. 2016, 52, 12262-12277.

(4) Zhu, M.; Zhou, H. Azobenzene-based small molecular photoswitches for protein modulation. Org. Biomol. Chem. 2018, 16, $8434-8445$

(5) Hüll, K.; Morstein, J.; Trauner, D. In vivo photopharmacology. Chem. Rev. 2018, 118, 10710-10747.

(6) Ube, T.; Kawasaki, K.; Ikeda, T. Photomobile liquid-crystalline elastomers with rearrangeable networks. Adv. Mater. 2016, 28, 82128217.
(7) Dong, L.; Feng, Y.; Wang, L.; Feng, W. Azobenzene-based solar thermal fuels: design, properties, and applications. Chem. Soc. Rev. 2018, 47, 7339-7368.

(8) Photoactive Functional Soft Materials: Preparation, Properties, and Applications; Li, Q., Ed.; Wiley-VCH: Weinheim, 2019.

(9) Oscurato, S. L.; Salvatore, M.; Maddalena, P.; Ambrosio, A. From nanoscopic to macroscopic photo-driven motion in azobenzene-containing materials. Nanophotonics 2018, 7, 1387-1422.

(10) Rau, H. Photochemistry and Photophysics; CRC Press: Boca Raton, Fl, 1990.

(11) Norikane, Y.; Kitamoto, K.; Tamaoki, N. Novel crystal structure, cis-trans isomerization, and host property of metasubstituted macrocyclic azobenzenes with the shortest linkers. J. Org. Chem. 2003, 68, 8291-8304.

(12) Nagamani, S. A.; Noirikane, Y.; Tamaoki, N. Photoinduced hinge-like molecular motion: studies on xanthene-based cyclic azobenzene dimers. J. Org. Chem. 2005, 70, 9304-9313.

(13) Garcia-Amorós, J.; Velasco, D. Recent advances towards azobenzene-based light-driven real-time information-transmitting materials. Beilstein J. Org. Chem. 2012, 8, 1003-1017.

(14) Garcia-Amorós, J.; Massad, W. A.; Nonell, S.; Velasco, D. Fast isomerizing methyl iodide azopyridinium salts for molecular switches. Org. Lett. 2010, 12, 3514-3517.

(15) Garcia-Amorós, J.; Nonell, S.; Velasco, D. Photo-driven optical oscillators in the $\mathrm{kHz}$ range based on push-pull hydroxyazopyridines. Chem. Commun. 2011, 47, 4022-4024.

(16) Garcia-Amorós, J.; Nonell, S.; Velasco, D. Light-controlled real time information transmitting systems based on nanosecond thermally-isomerising amino-azopyridinium salts. Chem. Commun. 2012, 48, 3421-3423.

(17) Garcia-Amorós, J.; Díaz-Lobo, M.; Nonell, S.; Velasco, D. Fastest thermal isomerization of an azobenzene for nanosecond photoswitching applications under physiological conditions. Angew. Chem., Int. Ed. 2012, 51, 12820-12823.

(18) Garcia-Amorós, J.; Bucinskas, A.; Reig, M.; Nonell, S.; Velasco, D. Fastest molecular photochromic switches based on nanosecond isomerizing benzothiazolium azophenolic salts. J. Mater. Chem. C 2014, 2, 474-480.

(19) Garcia-Amorós, J.; Reig, M.; Castro, M. C. R.; Cuadrado, A.; Raposo, M. M. M.; Velasco, D. Molecular photo-oscillators based on highly accelerated heterocyclic azo dyes in nematic liquid crystals. Chem. Commun. 2014, 50, 6704-6706.

(20) Simeth, N. A.; Crespi, S.; Fagnoni, M.; König, B. Tuning the thermal isomerization of phenylazoindole photoswitches from days to nanoseconds. J. Am. Chem. Soc. 2018, 140, 2940-2946.

(21) Crespi, S.; Simeth, N. A.; König, B. Heteroaryl azo dyes as molecular photoswitches. Nat. Rev. Chem. 2019, 3, 133-146.

(22) Qian, H.; Wang, Y.-Y.; Guo, D.-S.; Aprahamian, I. Controlling the isomerization rate of an Azo- $\mathrm{BF}_{2}$ switch using aggregation. J. Am. Chem. Soc. 2017, 139, 1037-1040.

(23) Onai, Y.; Mamiya, M.; Kiyokawa, T.; Okuwa, K.; Kobayashi, M.; Shinohara, H.; Sato, H. Colored merocyanine aggregates: longlived crystals of large size $(10-100 \mu \mathrm{m})$ and deaggregation of small aggregates in solutions. J. Phys. Chem. A. 1993, 97, 9499-9505.

(24) Fracaroli, A. M.; Granados, A. M.; de Rossi, R. H. E-Z isomerization and aggregation phenomena of dithiafulvenes in $\mathrm{CHCl}_{3}$. J. Org. Chem. 2009, 74, 2114-2119.

(25) Garcia-Amorós, J.; Castro, M. C. R.; Coelho, P.; Raposo, M. M. M.; Velasco, D. New heterocyclic systems to afford microsecond green-light isomerisable azo dyes and their use as fast molecular photochromic switches. Chem. Commun. 2013, 49, 11427-11429. 